\title{
Coupling of Reactive Power Planning for Operation and Voltage Stability Enhancement
}

\author{
Stefanie Samaan, Spyridon Iason Dizes, Markus Knittel, Albert Moser \\ Institute for Electrical Equipment \& Grids, Digitalisation \& Energy Economics \\ RWTH Aachen University \\ Aachen, Germany \\ s.samaan@iaew.rwth-aachen.de
}

\begin{abstract}
The ongoing decommissioning of conventional power plants decreases the installed reactive power reserves for voltage control in transmission grids. Hence, an efficient planning of compensation devices substituting this lack of reactive power is required. Grid operators must allocate these devices for steady-state voltage control and for dynamic voltage control ensuring voltage stability. A separate determination of this static and dynamic VAR demand, however, fails to exploit synergies and disregards that VAR compensation in steadystate reduces the reserves for dynamic compensation. This paper proposes a coupled determination of the system static and dynamic VAR demand. An optimisation method applying mixed-integer programming identifies an efficient allocation and portfolio consisting of different compensation technologies. It includes constraints for voltage limits during steady-state and contingencies as well as for long-term voltage stability. Results emphasise that the method identifies an efficient portfolio for various operation and fault scenarios, while providing the required voltage stability margin.
\end{abstract}

Keywords-long-term voltage stability, mixed-integer programming, reactive power compensation, steady-state VAR demand

\section{INTRODUCTION}

Transmission grid operators must ensure stable system voltages during operation and in case the system is subjected to contingencies. In transmission grids, both static and dynamic VAR reserves for voltage control are being diminished for reasons such as the decommissioning of conventional power plants and the higher utilisation of transmission lines. Different reactive power compensation technologies have to be considered to derive an efficient substitution for the decommissioned power plants. To adhere to the voltage limits in steady-state operation, passive compensation devices, in particular mechanically switched capacitors and reactors (MSCs / MSRs), can be installed in the grid. Compared to more advanced technologies, such devices require lower investment costs. However, the reactive power injection can be controlled only in discrete steps and it depends quadratically on the voltage at the point of common coupling. Thus, in case of a rapid voltage drop, MSCs are not sufficient for voltage control, as they further increase the voltage gradient. Active compensation devices, such as STATCOMs and synchronous condensers, on the other hand, allow a fast and continuous voltage control at higher investment costs. The proposed approach for reactive power planning (RPP) considers the allocation and technology selection of these compensation devices. It aims to determine the static and dynamic VAR demand for voltage control and to identify an efficient portfolio at minimum investment costs.

In recent research, several methods for RPP have been proposed. A common approach is the identification of the dynamic VAR demand to enhance short-term voltage stability. For this purpose, lower voltage limits for the voltage recovery after the fault clearing are defined in planning principles established by organisations developing critieria for reliable and secure grid operation such as the Western Electricity Coordinating Council (WECC) [1], [2]. An optimisation-based approach identifies the demand for dynamic VAR compensation to keep the voltage above these limits. To evaluate the effectiveness of a dynamic VAR compensation at different system buses, trajectory sensitivities can be applied [3], [4]. These time-dependent voltage sensitivities of the reactive power injection at the candidate buses are determined by means of time domain simulations. Therewith, it is possible to identify the most efficient buses to install compensation devices. Apart from the short-term voltage stability, the long-term voltage stability can be taken into consideration. One option would be to apply stability indices based on steady-state information to quantify the stabilising effects of VAR compensation [5], [6]. The indices identify the distance to the maximum loadability of the system. Furthermore, a sufficient voltage stability margin can be defined as a requirement in RPP [7]. According to the Western Systems Coordinating Council (WSCC), a MW loading margin of at least $5 \%$ within the base case and within (N-1)-conditions should be implemented as planning principles [6]. PV methods are one option to derive and further analyse the loading margin.

Various compensation devices can be used to fulfil the different requirements imposed by RPP. If the requirements depend on a specific reaction time or controllability, the technology characteristics must be considered to identify the a sufficient portfolio [1], [6]. While most approaches focus on the enhancement of voltage stability, requirements regarding voltage control in operation are barely taken into account. If operational requirements, such as voltage limits or generator reactive power capability limits, are considered, they are not coupled with the provision of a dynamic VAR reserve to ensure voltage stability [5], [8]. Within RPP, there is a shortage of approaches considering the planning aspects for the operational voltage control and for voltage stability enhancement simultaneously. Hence, the provision of dynamic VAR reserves for voltage control during contingencies and for providing a sufficient stability reserve is not coupled with the determination of steady-state VAR demand for operation. Thus, synergies remain unexploited and the identification of an efficient share of passive and active compensation devices within a portfolio is disregarded.

This paper proposes a method that enables the coupled determination of the static VAR demand for maintaining the operational voltage limits and the dynamic VAR demand for stabilising the voltage during contingencies and for providing a sufficient stability reserve. An optimisation algorithm based on mixed-integer linear programming (MILP) is applied to 
identify a portfolio satisfying the requirements from both operational voltage control and dynamic VAR demand. Thus, a portfolio for multiple scenarios regarding voltage control is identified and synergies are utilised to decrease the investment costs. As long as a sufficient dynamic VAR reserve is ensured, remaining VAR reserves can be used to control the voltage during steady-state operation. In order to derive a robust portfolio, characteristic grid use cases (GUCs) as well as critical contingencies are identified and simultaneously considered in the optimisation. A simplied test system is applied to introduce the proposed method.

\section{Multi-Stage APPROACH FOR RPP}

Only a robust portfolio of reactive power compensation devices allows sufficient voltage control in various operation and fault scenarios. The investigation of one single grid use case is neither adequate nor representative for the determination of a robust portfolio. Instead, an hourly time series for the planning year could be taken into account although this also significantly increases the size of the planning problem. In this paper, we identify characteristic GUCs by means of the generation and load pattern. Within these GUCs, the static VAR demand adjusting the voltages to adhere to the operational limits is identified. When determining the dynamic VAR demand, two aspects are considered. First, the dynamic VAR demand for voltage control in case of an outage is analysed. For this purpose, $(\mathrm{N}$ 1)-faults are evaluated on the basis of their impact on the operational voltage profile. The outages leading to the highest violations of either the upper or the lower voltage limits are selected for RPP. Second, the dynamic VAR demand to ensure a sufficient voltage stability margin for each investigated GUC is identified.

The described determination of the static and dynamic VAR demand including the portfolio identification consists of two stages as depicted in Fig. 1. Within the first stage, the static VAR demand to maintain the voltage within the operational limits is identified. All operational voltage-limit violations are compensated on the basis of the first-stage results.

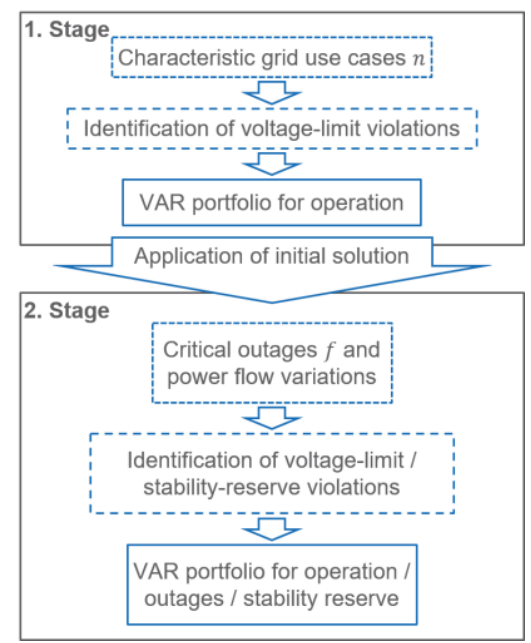

Fig. 1. Multi-stage approach for RPP.

Applying the derived portfolio and the corresponding VAR injections as an initial solution, the second stage investigates the dynamic VAR demand. This demands arises from voltage-limit violations due to the $(\mathrm{N}-1)$-faults and from requirements for voltage stability margins. During this stage, the operational voltage limits are further considered as requirements. As a result, the portfolio derived from the first stage can be substituted by a more efficient one, taking into account both the static and the dynamic VAR demand.

\section{A. 1. Stage: Static VAR Demand in Operation}

Voltage limits that must be adhered to during operation are defined in the grid planning principles. Within the planning principles of the German Transmission System Operators (TSOs), the voltage limits of the transmission voltage levels are defined as $V_{\text {lower }}^{380 \mathrm{kV}}=390 \mathrm{kV} / V_{\text {lower }}^{220 \mathrm{kV}}=220 \mathrm{kV}$ and $V_{\text {upper }}^{380 \mathrm{kV}}=420 \mathrm{kV} / V_{\text {upper }}^{220 \mathrm{kV}}=245 \mathrm{kV}$ respectively [9]. Reactive power must be provided during operation to ensure a voltage profile within these limits. Depending on the GUC, an inductive or capacitive VAR demand can occur. For the operating points of the devices, a distinction is made between overexcited (capacitive) and underexcited (inductive) operation. MSRs and MSCs are efficient technologies for compensating the operational VAR demand. In addition, STATCOMs and synchronous condensers can contribute to the operational voltage control. However, these active devices must also ensure a sufficient dynamic VAR reserve to enhance the voltage stability and to control the system voltages in case of contingencies.

Within the applied optimisation approach, the RPP objective is the minimisation of the fixed $c^{C A P E X}$ and variable $c^{O P E X}$ VAR costs. This approach considers different GUCs with diverse VAR demands to identify a robust portfolio. The GUCs are coupled by means of binary optimisation variables representing the installation decision at each candidate bus. Each technology is represented with one binary variable per candidate bus. If the binary variable of a candidate bus equals one, the rated power of the corresponding device is available in all GUCs. The VAR compensation of these installed devices is determined separately for each GUC by means of continuous optimisation variables. A common approach is the coupling of the binary and continuous variables in the objective function. This coupling results in a non-linear objective function and averts the application of optimisation strategies for MILP. To avoid this non-linearity in this approach, the binary and continuous optimisation variables are not coupled within the target function and are only multiplied by the respective cost terms:

$$
\begin{aligned}
& \min F\left(\vec{x}, \vec{Q}_{n}\right)= \\
& c_{M S C}^{C A P E X} \cdot \vec{x}_{M S C}+c_{M S R}^{C A P E X} \cdot \vec{x}_{M S R}+c_{S T A T}^{C A P E X} \vec{x}_{S T A T} \cdots \\
& \sum_{n}^{N_{G U C}} c_{M S C}^{O P E X} \cdot \vec{Q}_{n, M S C}^{O p}-c_{M S R}^{O P E X} \cdot \vec{Q}_{n, M S R}^{O p}+c_{S T A T}^{O P E X} \cdot \vec{Q}_{n, S T A T}^{O p}
\end{aligned}
$$

In this study, for simplification only STATCOMs represent the technology of active compensation devices. However, the optimisation problem can be easily extended with other active technologies, such as synchronous condensers. The binary variables that represent the general installation decision of the corresponding technologies are $\vec{x}_{M S C} \in[0,1], \vec{x}_{M S R} \in[0,1]$ and $\vec{x}_{S T A T} \in[0,1]$. Within one GUC $n$, the continuous variables representing the VAR compensation of the devices are $\vec{Q}_{n, M S C}^{O p}, \vec{Q}_{n, M S R}^{O p}$ and $\vec{Q}_{n, S T A T}^{O p}$. The upper and lower limits of the continuous variables depend on the rated power of the devices to be installed (6). A variation of the rated power of the devices can be applied to investigate the impact on the resulting portfolio. Within this study, the rated power of the devices is set to the commonly applied size of 300 Mvar. If the static and dynamic reactive 
power demand is less than this defined rated power, the size of the device could be reduced. The rated power of the devices $Q_{M S C}^{\max }, Q_{M S R}^{\max }, Q_{S T A T}^{\max }$ and $Q_{S T A T}^{\min }$ is multiplied with the corresponding binary variable to define the upper and lower limits of the continuous variables. Therewith, the continuous and binary variables are coupled by the constraints instead of the objective function:

$$
\left[\begin{array}{c}
0 \\
Q_{M S R}^{\max } \cdot \vec{x}_{M S R} \\
0 \\
Q_{S T A T}^{\min } \cdot \vec{x}_{S T A T}
\end{array}\right] \leq\left[\begin{array}{c}
\vec{Q}_{n, M S C}^{O p} \\
\vec{Q}_{n, M S R}^{O p} \\
\vec{Q}_{n, S T A T C}^{O p} \\
\vec{Q}_{n, S T A T u}^{O p}
\end{array}\right] \leq\left[\begin{array}{c}
Q_{M S C}^{\max } \cdot \vec{x}_{M S C} \\
0 \\
Q_{S T A T}^{\max } \cdot \vec{x}_{S T A T} \\
0
\end{array}\right]
$$

With regard to the VAR injection of the STATCOMs, a distinction is made between an underexcited $\vec{Q}_{n, S T A T u}^{O p}$ and an overexcited $\vec{Q}_{n, S T A T o}^{O p}$ operating point. The two continuous variables are coupled via one binary variable as described above. The impact of the devices' VAR injection on the voltage profile is determined by means of voltage-reactive power $(V-Q)$ sensitivities derived from the system Jacobian $J$ defined as follows:

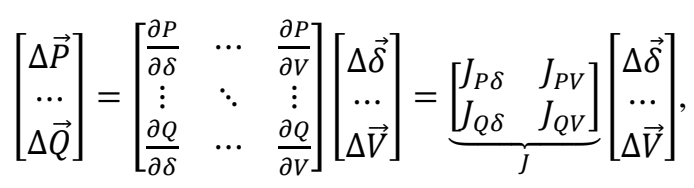

where $\Delta \vec{P}$ and $\Delta \vec{Q}$ are incremental changes in bus active and reactive power injection, and $\Delta \vec{\delta}$ and $\Delta \vec{V}$ are incremental changes in bus voltage angle and magnitude. The system Jacobian results from the power flow calculation of a GUC. One option to calculate the $V-Q$ sensitivities is the application of the reduced Jacobian matrix defined as:

$$
J_{R Q V}=J_{Q V}-J_{Q \delta} \cdot\left(J_{P \delta}\right)^{-1} \cdot J_{P V},
$$

under the assumption that $\Delta \vec{P}=0$ and $J_{P \delta}$ non-singular. The inverse of this reduced Jacobian matrix describes the impact of a bus VAR injection on all bus voltages of the system and therewith presents the $V-Q$ sensitivities $S^{\partial V / \partial Q}$ as derived with

$$
\Delta \vec{V}=\left(J_{R Q V}\right)^{-1} \cdot \Delta \vec{Q}=S^{\partial V / \partial Q} \cdot \Delta \vec{Q} .
$$

For large systems, LU decomposition is recommended for calculating the inverse matrices. For each GUC subjected to voltage-limit violations, the initial voltage profile $\vec{V}_{0}$ is adjusted by means of a static VAR injection. The voltage limits of all considered buses are added to the constraints of the MILP and concatenated with the continuous variables of the static VAR compensation and the $V-Q$ sensitivities as follows:

$$
\vec{V}^{\min } \leq \vec{V}_{0}+S_{0}^{\partial V / \partial Q} \cdot \Delta \vec{Q} \leq \vec{V}^{\max } .
$$

\section{B. 2. Stage: Static VAR Demand in Operation}

The second stage additionally takes into account the dynamic VAR demand required for voltage control during (N1)-faults as well as for the provision of a voltage stability reserve. Before determining the dynamic VAR demand, the results from the first stage are applied to eliminate the operational voltage-limit violations. In the second stage, the installation decision and the corresponding VAR injections resulting from the first stage can be adjusted, if a more efficient solution can be identified including both the static and the dynamic VAR demand. Assume that in the first stage a device is placed $\left(x_{i}^{1}=1\right)$ at bus $i$ with a VAR injection in GUC $n$ of $\Delta Q_{i, n}^{O p 1}$. In the second stage, the installation decision for this device can either be confirmed or reversed. If the installation decision is confirmed $\left(x_{i}^{2}=1\right)$, within the installation costs it must be taken into account that this decision is more cost-efficient than placing a device at a second bus $j$. In addition, the available VAR compensation in the second stage is reduced by the VAR injection identified in the first stage. If an installation decision is reversed $\left(x_{i}^{2}=0\right)$, the cost reduction must be considered within the objective function. In addition, it must be taken into account that the VAR injection resulting from the first stage is no longer available and must be substituted. Regarding the second-stage objective function, only the fixed costs require an adjustment depending on the installation decisions of the first stage. The fixed costs are adjusted with

$$
\begin{aligned}
& \text { case 1: } x_{i}^{1}=1 \rightarrow c_{i}^{\text {CAPEX2 }}=1 \cdot c_{i}^{\text {CAPEX1 }}, \\
& \text { case 2: } x_{i}^{1}=0 \rightarrow c_{i}^{\text {CAPEX2 }}=2 \cdot c_{i}^{\text {CAPEX1 }},
\end{aligned}
$$

and replace the fixed costs in equation (1). The constraints with respect to the acquirable VAR compensation of the second stage $\vec{Q}_{n}^{O p 2}$ are adjusted according to the VAR injections of the first step $\vec{Q}_{n}^{O p 1}$ as follows:

$\left[\begin{array}{c}0 \\ Q_{M S R}^{\max } \cdot \vec{x}_{M S R} \\ 0 \\ Q_{S T A T}^{\min } \cdot \vec{x}_{S T A T}\end{array}\right] \leq\left[\begin{array}{c}\vec{Q}_{n, M S C}^{O p 2}+\vec{Q}_{n, M S C}^{O p 1} \\ \vec{Q}_{n, M S R}^{O p 2}+\vec{Q}_{n, M S R}^{O p 1} \\ \vec{Q}_{n, S T A T o}^{O p 2}+\vec{Q}_{n, S T A T o}^{O p 1} \\ \vec{Q}_{n, S T A T u}^{O p 2}+\vec{Q}_{n, S T A T u}^{O p 1}\end{array}\right] \leq\left[\begin{array}{c}Q_{M S C}^{\max } \cdot \vec{x}_{M S C} \\ 0 \\ Q_{S T A T}^{\max } \cdot \vec{x}_{S T A T} \\ 0\end{array}\right]$.

If the installation decision at bus $i$ is reversed in the second stage $\left(x_{i}^{1}=1 \& x_{i}^{2}=0\right)$, the continuous variable of the VAR compensation takes the opposite value of the VAR injection of the first stage $\vec{Q}_{i, n}^{O p 2}=-\vec{Q}_{i, n}^{O p 1}$.

\section{2. Stage: Dynamic VAR Demand Caused by Outages}

The outage of a transmission system asset or a generation unit can lead to an increased voltage or, as in most cases, to a rapid voltage drop. For these scenarios, the planning principles define adjusted lower voltage limits. The German TSOs specify the limits $V_{\text {lower }}^{380 \mathrm{kV}}=380 \mathrm{kV}$ and $V_{\text {lower }}^{220 \mathrm{kV}}=$ $210 \mathrm{kV}$ as requirements for the voltage control during (N-1)faults. Only if sufficient dynamic VAR reserves have been kept, active compensation devices can ensure fast voltage control in case of voltage-limit violations. Therefore, the dynamic VAR demand $\vec{Q}_{n}^{(N-1)}$ caused by an outage in GUC $n$ is coupled with the static VAR injection for operational voltage control $\vec{Q}_{n}^{O p}$ of the corresponding GUC as follows:

$$
\begin{aligned}
& {\left[\begin{array}{c}
0 \\
Q_{S T A T}^{\min } \cdot \vec{x}_{S T A T}-\vec{Q}_{n, S T A T u}^{\text {Op1 }}-\vec{Q}_{n, S T A T u}^{\text {Op2 }}
\end{array}\right] \leq\left[\begin{array}{l}
\vec{Q}_{n, S T A T o}^{(N-1)} \\
\vec{Q}_{n, S T A T u}^{(N-1)}
\end{array}\right] \leq} \\
& {\left[\begin{array}{c}
Q_{S T A T}^{\max } \cdot \vec{x}_{S T A T}-\vec{Q}_{n, S T A T o}^{\text {Op1 }}-\vec{Q}_{n, S T A T o}^{\text {Op2 }} \\
0
\end{array}\right] .}
\end{aligned}
$$

As only active devices allow fast voltage control, MSCs and MSRs are not considered for these fault scenarios. The adjusted lower voltage limits are included in the constraints. To derive robust V-Q sensitivities, the respective calculations are repeated on the basis of the power flow calculations in the (N-1)-scenarios including the VAR compensation resulting from the first stage. The upper and the lower voltage limits are 
concatenated with the continuous variables for the dynamic VAR compensation and these V-Q sensitivities. The resulting constraints can be written in the form:

$$
\vec{V}_{(N-1)}^{\min } \leq \vec{V}_{(N-1)}+S_{(N-1)}^{\partial V / \partial Q} \cdot \Delta \vec{Q} \leq \vec{V}^{\max }
$$

\section{2. Stage: Dynamic VAR Demand for Stability Reserve}

The RPP approach we present in this paper, requires a sufficient voltage stability margin, as described in planning principles applied by organisations such as WSCC. This margin is set to $15 \%$ with respect to the total system load of the corresponding GUC. For the analysis of this requirement, the selected GUCs are subjected to a variation in their power flow pattern. The unit vectors representing a change in generation and load pattern are defined by the intra-hour transition of the GUC (target case) and the hour before the corresponding GUC (base case). Thus, each GUC is subjected to a variation of the power flow pattern in this pre-defined direction in the size of $15 \%$ of the system load. For these analyses, the method of the continuation power flow is applied. As long as the bifurcation (instability) point not occurs within this $15 \%$ range, the GUC is stated as stable regarding long-term voltage stability. If the stability margin of at least $15 \%$ is not achieved, the optimisation identifies the required amount of dynamic VAR compensation for increasing this margin. For this purpose, the sensitivities of a system parameter change regarding the stability margin are determined at the bifurcation point of the P-V curve [11]. The determination of these sensitivities is based on an equilibrium satisfying the steady-state equations of the power system:

$$
F(x, p, \lambda)=0,
$$

where $x$ is the state vector and $\lambda$ a vector of load parameters. The vector $p$ describes power system parameters such as a line admittance or capacitor susceptance. For a given base and target scenario, the direction of the change in load or generation is specified with the according unit vectors:

$$
\begin{gathered}
k_{l}^{p}=\frac{P_{l}^{\text {target }}-P_{l}^{\text {base }}}{\left|P_{l}^{\text {target }}-P_{l}^{\text {base }}\right|}, k_{l}^{q}=\frac{Q_{l}^{\text {target }}-Q_{l}^{\text {base }}}{\left|Q_{l}^{\text {target }}-Q_{l}^{\text {base }}\right|}, \\
k_{g}^{p}=\frac{P_{g}^{\text {target }}-P_{g}^{\text {base }}}{\left|P_{g}^{\text {target }}-P_{g}^{\text {base }}\right|} .
\end{gathered}
$$

While $k_{l}^{p}$ and $k_{l}^{q}$ represent the active and reactive power load direction, $k_{g}^{p}$ represents the direction of the active power generation. A linear sensitivity of the stability margin with respect to a parameter change can be calculated at the bifurcation point $(*)$ with

$$
\frac{\partial M^{*}}{\partial p}=\frac{w^{*} F_{p}^{*}}{w^{*} F_{\lambda}^{*} k},
$$

where $w$ is the left eigenvector corresponding to the zero eigenvalue of the system Jacobian; $F_{\lambda}^{*}$ is the derivative of $F$ with respect to the load parameter and $F_{p}^{*}$ is the derivative with respect to any system parameter $p$. The change of the stability margin with respect to a system parameter change can be estimated with these sensitivities as described in the following equation:

$$
\Delta M=\frac{\partial M^{*}}{\partial p} \cdot \Delta p
$$

In this approach, these sensitivities are applied to identify the impact of a VAR injection on the stability margin. Therefore, the VAR demand emerging from the stability margin requirement $\vec{Q}_{n}^{\lambda}$ is coupled with the static VAR demand for operational voltage control $\vec{Q}_{n}^{O p}$ in the corresponding GUC via the constraints:

$$
\begin{aligned}
& {\left[\begin{array}{c}
0 \\
Q_{S T A T}^{\min } \cdot \vec{x}_{S T A T r}-\vec{Q}_{n, S T A T u}^{\text {Op1 }}-\vec{Q}_{n, S T A T u}^{\text {Op2 }}
\end{array}\right] \leq\left[\begin{array}{l}
\vec{Q}_{n, S T A T o}^{\lambda} \\
\vec{Q}_{n, S T A T u}^{\lambda}
\end{array}\right] \leq} \\
& {\left[\begin{array}{c}
Q_{S T A T}^{\max } \cdot \vec{x}_{S T A T}-\vec{Q}_{n, S T A T o}^{\text {op } 1}-\vec{Q}_{n, S T A T o}^{\text {Op } 2} \\
0
\end{array}\right],}
\end{aligned}
$$

The required stability margin $M_{\min }$ is added as a constraint to the MILP and concatenated with the continuous variables of the dynamic VAR compensation $\Delta \vec{Q}^{\lambda}$ as well as the respective stability margin sensitivities as follows:

$$
M_{\min } \leq M_{0}+\frac{\partial M^{*}}{\partial Q} \cdot \Delta \vec{Q}^{\lambda}
$$

At this point it should be emphasized that the stability margin is a system wide quantity and valid for one operation point in combination with the unit vectors representing the variation in generation and load pattern. Thus, the initial and the required stability margins unlike the initial and the required voltage profiles are scalar. The stability margin sensitivities are vectorised.

\section{CASE STUDY}

The assessment of the proposed RPP approach is performed with a modified IEEE 9-bus system as shown in Fig. 2. This system allows a simplified analysis of the method introduced. The transmission system is represented by six buses in the $380 \mathrm{kV}$ level. Compared to the conventional IEEE 9-bus system, the line length has been increased, thus leading to higher VAR losses. One synchronous generator is connected to the system via a $27 \mathrm{kV} / 380 \mathrm{kV}$ transformer equipped with a tap changer. This generator represents the only voltage-controlled bus in the system. The other two synchronous generators of the conventional IEEE 9-bus system are replaced by converter-coupled generator units based on renewable energy systems with a power factor of $\cos (\varphi)=0.928$. These generation units as well as the three constant power loads are coupled with the transmission system via $110 \mathrm{kV} / 380 \mathrm{kV}$ transformers.

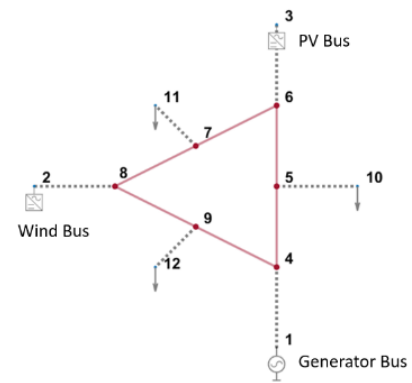

Fig. 2. Topology of modified IEEE 9-bus system

As mentioned above, the identification of a robust portfolio requires the assessment of different GUCs. Therefore, the IEEE 9-bus system is extended by an annual time series with an hourly resolution. While the two convertercoupled generation units are equipped with one photovoltaicand one wind-generation time series, the conventional generator is utilised for balancing the system load. The maximum generation share of the renewables amounts to $15 \%$. All three loads are equipped with the same hourly time series, though they are scaled differently. The annual peak 
load amounts to $900 \mathrm{MW}$. For the application of the planning approach, 40 GUCs are identified on the basis of their load and generation pattern.

\section{A. 1. Stage: Static VAR Demand in Operation}

The first stage identifies the static VAR demand in the 40 GUCs due to violations of the lower and upper voltage limits. Since, the test system is characterised by a significant lack of reactive power, voltage-limit violations occur in every of the assessed GUCs. The resulting portfolio consists of two MSCs (bus $6 \&$ bus 7) and one MSR (bus 4). Fig. 3 illustrates the voltage profile of the system in an exemplary GUC both with and without the VAR compensation resulting from the first stage of the optimisation. The load of the GUC amounts to 783 MW, which is about $87 \%$ of the annual peak load. It can be observed that the compensated voltage profile still is violating the lower voltage limit. In contrast, the forecasted voltage profile calculated on the basis of the V-Q sensitivities and the resulting VAR compensation adheres to the voltage limits. This emphasizes that the linearised V-Q sensitivities can only approximate the impact of VAR compensation. Thus, a second step needs to be performed to eliminate the deviations between the forecasted voltage profile and the voltage profile resulting from power flow calculations.

\section{B. 2. Stage: Static VAR Demand in Operation}

To perform the proposed step for correcting the remaining voltage-limit violations of the first stage, the MILP described in the second stage of the approach is applied. As proven in Fig. 3, this step derives a sufficient VAR compensation leading to a voltage profile within the lower and upper voltage limits. While the first step identifies a VAR demand of 138 Mvar in the exemplary GUC, the second step corrects this demand to 213 Mvar. Yet, the number and allocation of the compensation devices is not adjusted.

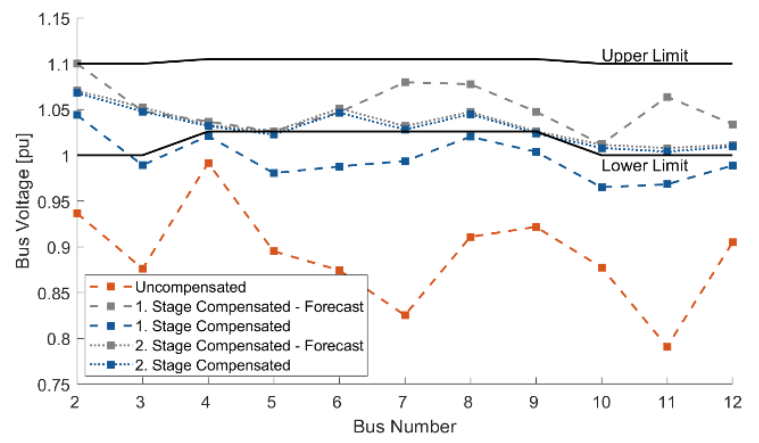

Fig. 3. Exemplary voltage profile at PQ buses compensated with VAR portfolio resulting from first / second stage.

Fig. 4 compares the VAR supply of the compensation devices based on the results of the first and second stage. In addition, the transmission system VAR demand is illustrated.

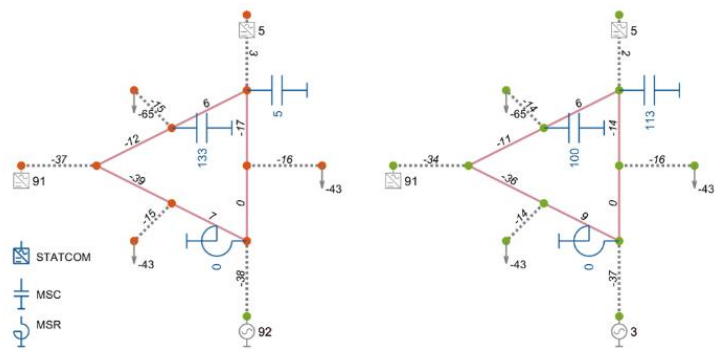

Fig. 4. Comparison of reactive power compensation and demand [Mvar] resulting from first and second stage.
The uncompensated and compensated voltage profiles of all 40 analysed GUCs are summarised in Fig. 5. Only in some GUCs the violations of the lower voltage limit are not eliminated with the VAR compensation resulting from the second stage. In this case, another correction step could be applied.

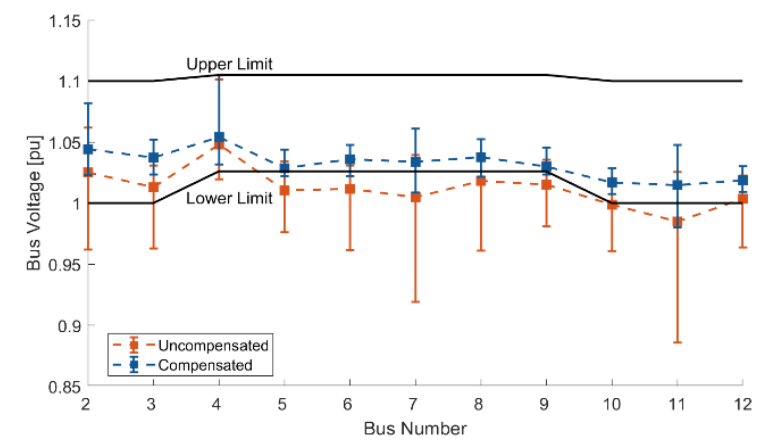

Fig. 5. Summarised voltage profile at PQ buses compensated with VAR portfolio resulting from second stage.

\section{2. Stage: Dynamic VAR Demand Caused by Outages}

In general, the most severe outages should be considered when determining the dynamic VAR demand caused by $(\mathrm{N}$ 1)-faults. However, within the test system the number of $(\mathrm{N}-$ 1)-faults to be analysed is limited by the non-convergence resulting from most outages. For this reason only in 19 of 40 GUCs between one and three (N-1)-faults are analysed. The portfolio resulting from the optimisation introduced in section II C consists of one MSC (bus 6) and two STATCOMs (bus 4 and bus 7). Only the STATCOMs controls the voltage profile after the system has been subjected to (N-1)-faults by means of an adjusted operating point. Fig. 6 illustrates an exemplary voltage profile without and with the VAR compensation of the derived portfolio. The applied (N-1)-fault leads to the outage of the line connecting bus 5 and bus 6 . The two STATCOMs inject sufficient VAR to adhere to the voltage limits valid during the (N-1)-fault.

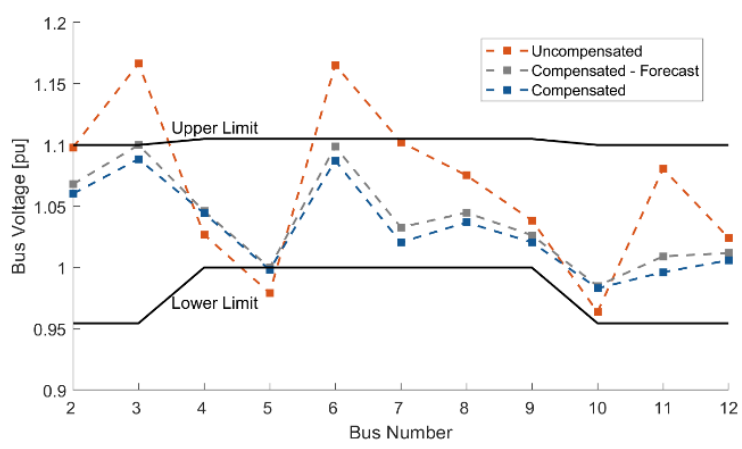

Fig. 6. Exemplary voltage profile at PQ buses for a (N-1)-fault compensated with VAR portfolio resulting from second stage.

\section{2. Stage: Dynamic VAR Demand for Stability Reserve}

To analyse the dynamic VAR demand ensuring a sufficient voltage stability margin, in this study a margin of at least $15 \%$ is required. Most GUCs fulfil this requirement without any additional VAR compensation devices. In six GUCs this required stability margin is not provided. The portfolio resulting from the optimisation introduced in section II D consists of one MSC (bus 7), one MSR (bus 4) as well as one STATCOM (bus 5). Fig. 7 emphasises the impact of the VAR compensation on a GUC with an initial base-case load of $893 \mathrm{MW}$. While the uncompensated GUC provides of 
stability margin of $11.5 \%$, an additional VAR injection of 50 Mvar increases this margin to $14.7 \%$.

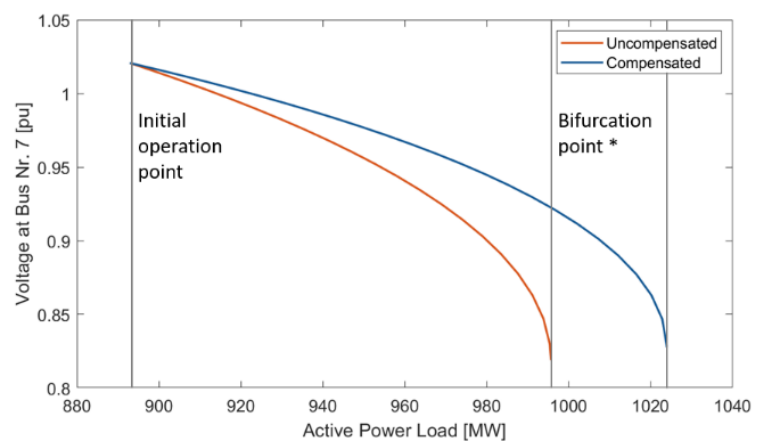

Fig. 7. Exemplary PV curve compensated with VAR portfolio resulting from second stage.

\section{E. Resulting Portfolios}

Fig. 8 summarises the portfolios resulting from the operational VAR demand in the first and second stage (a) as well as the consideration of the required stability margin (b) and the outages (c). The final portfolio considers the static and dynamic VAR demand of all assessed GUCs including both the required stability margin and the outages (d). Since, the dynamic VAR demand can only be provided by active devices, the final portfolio has to contain STATCOMs. The STATCOMs allow an underexcited operation for controlling the operational voltage profile, as well as an overexcited operation for the provision of the required voltage stability margin. Thus, the technology of the MSR is substituted.

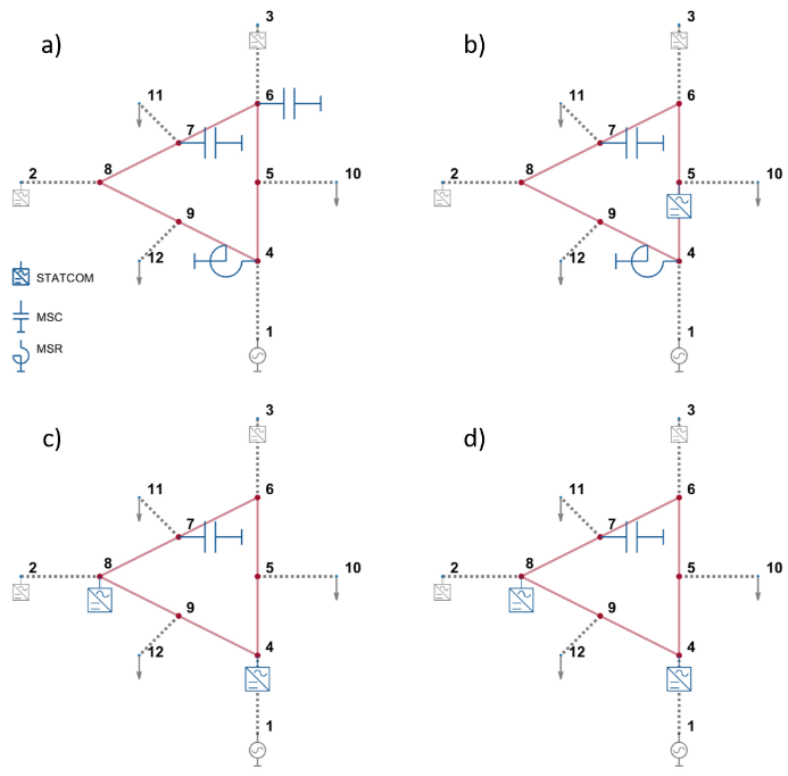

Fig. 8. Resulting portfolio of stage 1 and 2 a), with consideration of stability margin $b$ ), with consideration of outages $c$ ), with consideration of stability margin and outages d).

The maximum VAR injections of the compensation technologies resulting from the different optimisations are summerised in Table I. It can be seen that the devices' rated reactive power of 300 Mvars is not fully required in any scenario. However, a smaller rated power of for example 200 Mvars would not be sufficient for the RPP including the outages and the required stability margin.
TABLE I.

MAXIMUM REACTIVE POWER INJECTIONS

\begin{tabular}{|l|l|l|l|}
\hline \multirow{2}{*}{$\begin{array}{c}\text { Stage / } \\
\text { Scenario }\end{array}$} & \multicolumn{1}{|c|}{ Installed Technology: Bus, $\left(\boldsymbol{Q}_{\max }[\right.$ Mvar] $)$} \\
\cline { 2 - 4 } & \multicolumn{1}{|c|}{$\boldsymbol{M S C}$} & \multicolumn{1}{c|}{$\boldsymbol{M S R}$} & \multicolumn{1}{c|}{$\boldsymbol{S T A T C O M}$} \\
\hline 1. Stage / a) & $\begin{array}{l}6,(53) ; \\
7,(140)\end{array}$ & $4,(-123)$ & $/$ \\
\hline 2. Stage / a) & $\begin{array}{l}6,(126) ; \\
7,(170)\end{array}$ & $4,(-123)$ & $/$ \\
\hline 2. Stage / b) & $7,(234)$ & $4,(-123)$ & $5,(61)$ \\
\hline $\begin{array}{l}\text { 2. Stage / } \\
\text { c) / d) }\end{array}$ & $6,(275)$ & $/$ & $\begin{array}{l}4,(221,-122) ; \\
7,(167,-123)\end{array}$ \\
\hline
\end{tabular}

\section{CONCLUSION}

This paper proposes a coupled determination of the static and dynamic VAR demand for both voltage control during operation and contingencies as well as for the provision of the required stability margin. Therefore, a multi-stage optimisation based on MILP is introduced and applied to a test system. The results emphasize that this approach enables the identification of a robust portfolio for various voltage control requirements in different GUCs and fault scenarios. Since the installed active devices provide static and dynamic VAR compensation, the RPP utilises synergies efficiently. In future studies, we will apply this approach to complex grid topologies to validate the optimisation for real-scale systems and to prove its generality. In addition, the proposed approach should be extended by a more detailed presention of operational concepts as well as voltage-stability requirements to enhance short-term voltage stability and transient stability.

\section{REFERENCES}

[1] H. Liu, V. Krishnan, J. D. McCalley, and A. Chowdhury, "Optimal planning of static and dynamic reactive power resources," IET Generation, Transmission \& Distribution, vol. 8, no. 12, pp. 1916 1927, 2014.

[2] Y. Wang, H. Chen, and F. Li, Eds., "Reactive power planning with transient process stability constraint," 2015 IEEE Power \& Energy Society General Meeting, 2015.

[3] B. Sapkota and V. Vittal, "Dynamic VAr Planning in a Large Power System Using Trajectory Sensitivities," IEEE Transactions on Power Systems, vol. 25, no. 1, pp. 461-469, 2010.

[4] M. Paramasivam et al., Eds., "Dynamic optimization based reactive power planning to mitigate slow voltage recovery and short term voltage instability," 2014 IEEE PES General Meeting | Conference \& Exposition, 2014.

[5] Y. Xu, Z. Y. Dong, C. Xiao, R. Zhang, and K. P. Wong, "Optimal placement of static compensators for multi-objective voltage stability enhancement of power systems," IET Generation, Transmission \& Distribution, vol. 9, no. 15, pp. 2144-2151, 2015.

[6] A. M. Tahboub, M. S. E. Moursi, W. L. Woon, and J. L. Kirtley, "Multiobjective Dynamic VAR Planning Strategy With Different Shunt Compensation Technologies," IEEE Transactions on Power Systems, vol. 33, no. 3, pp. 2429-2439, 2018.

[7] V. Krishnan, H. Liu, and J. D. McCalley, Eds., "Coordinated reactive power planning against power system voltage instability," 2009 IEEE/PES Power Systems Conference and Exposition, 2009.

[8] A. M. Tahboub, S. Alaraifi, and M. S. Elmoursi, Eds., "Dynamic VAR planning for rotor-angle and short-term voltage stability enhancement, " 2017 IEEE International Conference on Industrial Technology (ICIT), 2017.

[9] 50Hertz Transmission GmbH and Amprion GmbH, TenneT TSO GmbH, TransnetBW GmbH, "Grundsätze für die Ausbauplanung des deutschen Übertragungsnetzes,” Jul. 2018.

[10] "Bewertung der Systemstabilität: Begleitdokument zum Netzentwicklungsplan Strom 2030," Version 2019, zweiter Entwurf," Stand Apr. 2019.

[11] S. Greene, I. Dobson, and F. L. Alvarado, "Sensitivity of the loading margin to voltage collapse with respect to arbitrary parameters," IEEE Transactions on Power Systems, vol. 12, no. 1, pp. 262-272, 1997. 